\title{
Mysterious chokeberries: new data on the diversity and phylogeny of Aronia Medik. (Rosaceae)
}

\author{
Alexey SHIPUNOV ${ }^{1, *}$, Sofia GLADKOVA ${ }^{2}$, Polina TIMOSHINA ${ }^{3}$, Hye Ji LEE ${ }^{4}$, \\ Jinhee $\mathrm{CHOI}^{5}$, Sarah DESPIEGELAERE ${ }^{5} \&$ Bryan CONNOLLY $^{5}$ \\ ${ }^{1,4,5,6}$ Minot State University, Biology, 500 University Ave, Minot, ND, USA. \\ ${ }^{2,3}$ Department of Biology, Moscow State University, Russia. \\ ${ }^{7}$ Framingham State University, Biology, 100 State St, Framingham, MA, USA. \\ *Corresponding author: dactylorhiza@gmail.com \\ 2Email:mgu@univer.msu.ru \\ ${ }^{3}$ Email:mgu@univer.msu.ru \\ ${ }^{4}$ Email:msu@minotstateu.edu \\ ${ }^{5}$ Email:msu@minotstateu.edu \\ ${ }^{6}$ Email: msu@minotstateu.edu \\ ${ }^{7}$ Email: bconnolly@framingham.edu
}

\begin{abstract}
Aronia Medik. (chokeberry, Rosaceae) is a genus of woody shrubs with two or three North American species. Species boundaries and relationships between species of Aronia are frequently under question. The only European species in the genus, A. mitschurinii A.K.Skvortsov \& Maitul., is suggested to be an inter-generic hybrid. In order to clarify the relationships between species of Aronia, we performed several morphometric and molecular analyses and found that the molecular and morphological diversity within data on American Aronia is low, and species boundaries are mostly not clearly expressed. Whereas morphology is able to separate American species from A. mitschurinii, there is no support for such discrimination from the molecular data; our analyses did not reveal evidence of A. mitschurinii hybrid origin. We believe that higher-resolution markers are needed to resolve species boundaries and putative hybridization events.
\end{abstract}

Keywords. Aronia, Rosaceae, DNA, morphology, hybridity.

Shipunov A., Gladkova S., Timoshina P., Lee H.J., Choi J., Despiegelaere S. \& Connolly B. 2019. Mysterious chokeberries: new data on the diversity and phylogeny of Aronia Medik. (Rosaceae). European Journal of Taxonomy 570: 1-14. https://doi.org/10.5852/ejt.2019.570

\section{Introduction}

Chokeberry, Aronia Medik. (Medikus 1789), is a genus of woody shrubs in the family Rosaceae. The boundaries of species of Aronia are unclear and typically three (Hardin 1973; Persson Hovmalm et al. 2004) or two (Robertson et al. 1991) species are recognized in North America. The following North American species are recognized:1) Aronia melanocarpa (Michx.) Elliott or black chokeberry (with 
diploid and tetraploid forms; Connolly 2014), 2) Aronia arbutifolia (L.) Pers. or red chokeberry, 3) Aronia $\times$ floribunda (Lindl.) Spach (synonyms: Aronia $\times$ prunifolia (Marshall) Rehder, Aronia atropurpurea Britton) or purple chokeberry (likely of hybrid origin with possible subsequent introgression; Connolly 2014). The only European species in the genus, A. mitschurinii (Skvortsov \& Majtulina 1982; Skvortsov et al. 1983) or Mitschurin's chokeberry is suggested to be an inter-generic hybrid $\times$ Sorbaronia mitschurinii (Skvortsov \& Maitul.) Sennikov, but its origin is not yet clarified (Leonard 2011; Smolik et al. 2011; Leonard et al. 2013; Sennikov \& Phipps 2013; Vinogradova \& Kuklina 2014).

Chokeberries are agriculturally promising for fruit and nutraceuticals, and also have applications as ornamental landscape plants (Brand 2010; Taheri et al. 2013; Connolly 2014). Aronia mitschurinii has bigger and tastier fruits then other aronias and was adopted as valuable agricultural fruit plant across North America (Kask 1987).

We believe that instability in Aronia taxonomy is largely the result of insufficient sampling; thus, our goal was to increase sampling, use expert knowledge to identify or re-identify our samples, and then to employ DNA sequence analyses and advanced morphometry methods in order to perform a comprehensive analysis of Aronia diversity which will clarify species relationships.

\section{Material and methods}

\section{DNA sequencing and molecular analysis}

We sequenced the nuclear rDNA internal transcribed spacer 2 (ITS2), the chloroplast encoded $r b c \mathrm{~L}$ gene and trnL-F spacer (Kuzmina \& Ivanova 2011) from several representatives of each of five Aronia species (34, 38 and 15, respectively). These markers are commonly used for studying the phylogeny and origins of angiosperms species including Rosaceae (Campbell et al. 2007; Li et al. 2012; Lo \& Donoghue 2012; Dluzewska et al. 2013; Zarrei et al. 2014). In addition, we amplified and sequenced the second intron of the nuclear low-copy LEAFY gene which has been considered a useful marker in phylogenetic analysis of Rosaceae (Oh \& Potter 2003; Lo et al. 2007; Li et al. 2017).

We obtained 42 tissue samples from herbarium collections in the USA (CAS, F, HUH, JEPS, MO, NY, UC, US) and Russia (MHA). We also used 31 freshly collected leaf tissue samples taken in the field and from the Rosaceae living collection of the University of Connecticut (USA). Field collected material (mostly of $A$. mitschurinii) was from the Central Russian Tverj and Moscow regions, where tissue samples were obtained from the living plants growing in the cultivation and the living plants that have escaped from the cultivation. The list of samples, all sequences, datasets and scripts are available on Zenodo (https://doi.org/10.5281/zenodo.3276157). All sequences were also submitted to GenBank.

In addition to Aronia, we used samples of Sorbus aucuparia L. (Linnaeus 1753) and of the intergeneric hybrid, $\times$ Sorbaronia fallax (C.K.Schneid.) C.K.Schneid. (Sorbus aucuparia $\times$ Aronia melanocarpa: Schneider 1906; Connolly 2009). GenBank sequences of $S$. aucuparia were also used. The first sample is phylogenetically distant from both Aronia and Sorbus s.str. (Sun et al. 2018) and therefore was used as an outgroup in most phylogenetic analyses. Considering problems with Aronia species identification (typical issue is that proper determination requires checking the autumn coloration of fruits: Hardin 1973), GenBank sequences of this genus were not thought to be reliably identified.

DNA was extracted using either a MO BIO PowerPlant DNA Isolation Kit (MO BIO Laboratories, Carlsbad, California, USA), or NUCLEOSPIN Plant II Kit (MACHEREY-NAGEL GmbH \& Co. KG, Düren, Germany). Dry plant leaf material (typically, 0.05-0.09 g) was powdered using a sterile mortar and pestle and then processed in accordance with the supplied protocol. We increased the lysis time to 30 minutes and used thermomixer on the slow rotation speed $(350 \mathrm{rpm})$ instead of water bath. 
Nanodrop 1000 Spectrophotometer (Thermo Scientific, Wilmington, DE, USA) was used to assess the concentration and purity (the 260/280 nm ratio of absorbance) of DNA samples.

We sequenced the markers mentioned above using primers and protocols in accordance with recommendations of the Barcoding of Life Consortium (Kuzmina \& Ivanova 2011) and the LFY1/LFY2 primers (Oh \& Potter 2003). PCR was carried out as follows: the reaction mixture in a total volume of $20 \mu \mathrm{L}$ contained $5.2 \mu \mathrm{L}$ of PCR Master Mix (components from QIAGEN Corporation, Germantown, Maryland), $1 \mu \mathrm{L}$ of $10 \mu \mathrm{M}$ forward and reverse primers, $1 \mu \mathrm{L}$ of DNA solution from the extraction above and $11.8 \mu \mathrm{L}$ purified water. Samples were incubated in a thermal cycler: $94^{\circ}$ for $5 \mathrm{~min}$, then 35 cycles of $94^{\circ}$ for $1 \mathrm{~min} ; 51^{\circ}$ for $1 \mathrm{~min}, 72^{\circ}$ for $2 \mathrm{~min}$, and finally $72^{\circ}$ for $10 \mathrm{~min}$. Regardless to the marker and species, we always received single band PCR products. They were sent for purification and sequencing to Functional Biosciences, Inc. (Madison, Wyoming) and sequenced there in accordance with standard protocol. Sequences were obtained, assembled and edited using Sequencher ${ }^{\mathrm{TM}} 4.5$ (Genes Codes Corporation, Ann Arbor, Michigan, USA) and then aligned with AliView / Muscle (Larsson

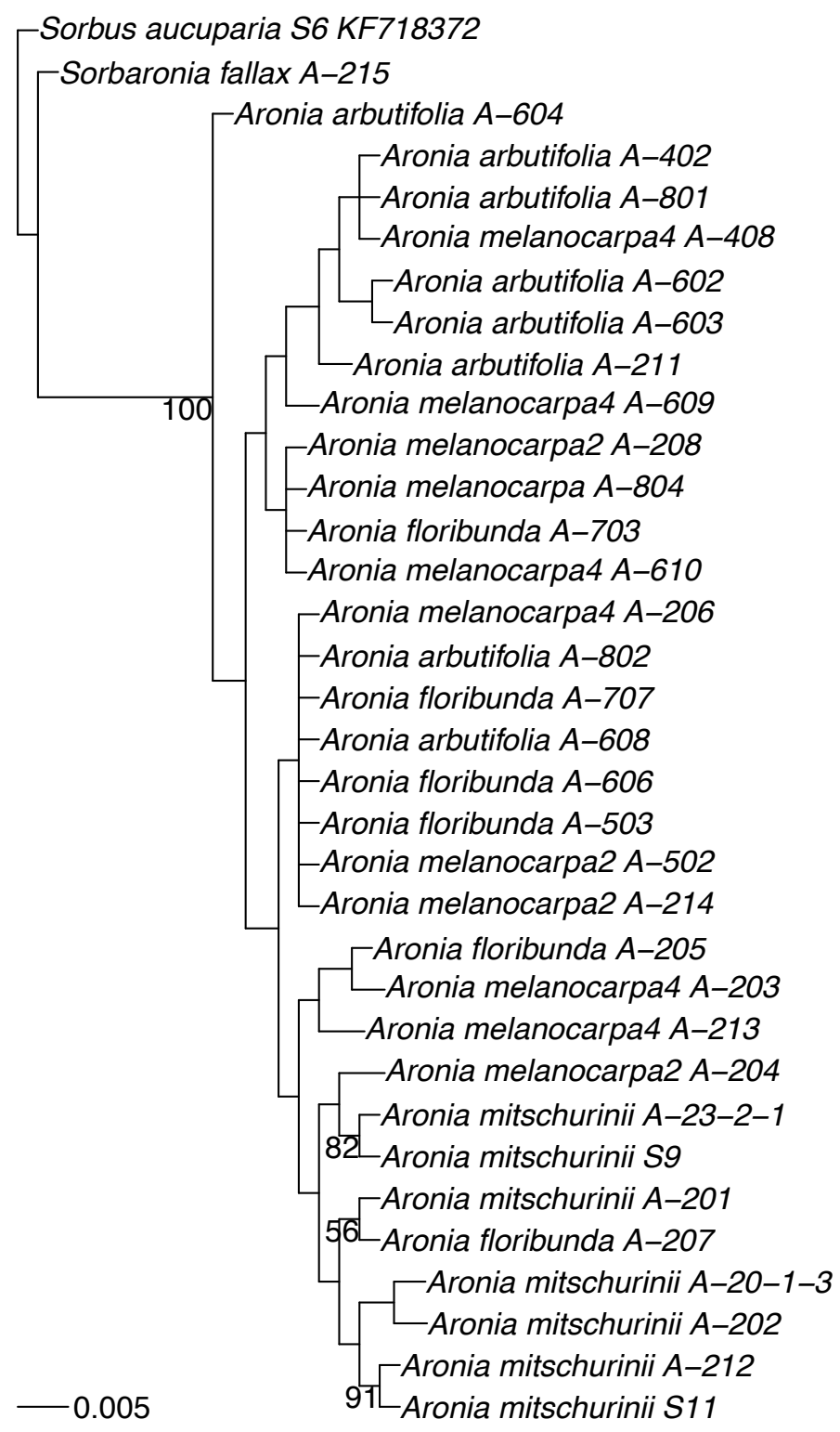

Fig. 1. ML tree resulted from the analysis of concatenated ITS2 and $r b c \mathrm{~L}$ sequences. 
2014) and ClustalX (Thompson et al. 1997) using gap opening cost 9 , gap extension cost 0.05 and IUB weight matrix.

In total, we produced 309 DNA sequences, and after the preliminary analysis, selected the 113 longest and minimally noisy sequences for further research. Those selected sequences belonged to 42 individual plants from all species of Aronia (12-24 sequences per species). In terms of the markers, most successful were the amplification and subsequent sequencing of $r b c \mathrm{~L}$ (35 sequences) and the least successful the amplification of the first variant of LEAFY second intron (6 sequences). Phylogenetic analyses were implemented with the help of the R ape and phangorn packages (Paradis et al. 2004; Schliep 2011). We used the Kimura distance, neighbor-joining and maximum likelihood (under a GTR model with gamma distribution selected using the Akaike information criterion implemented in R) trees for the most of our calculations.

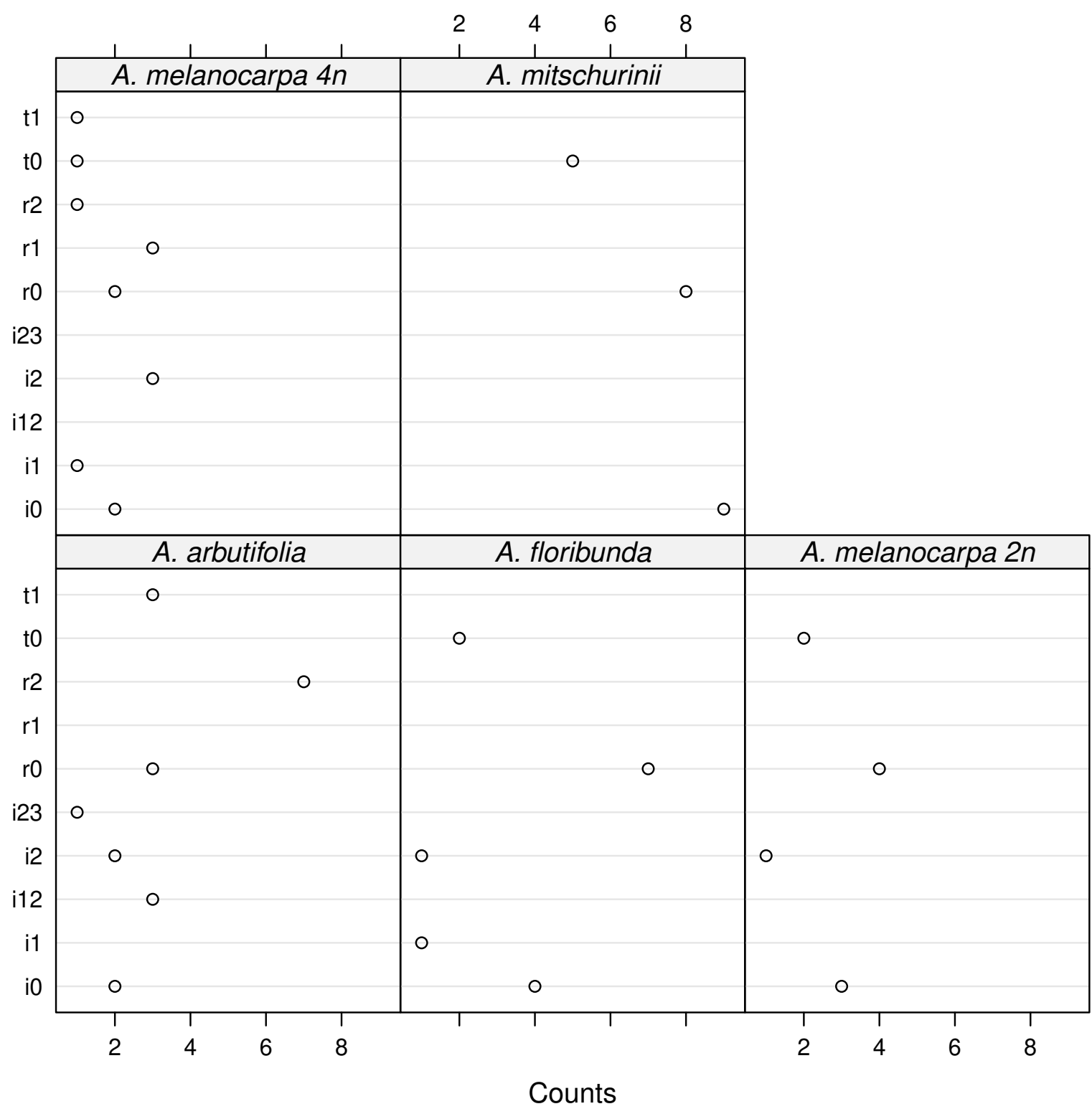

Fig. 2. Abundance of haplotypes per species. Each rectangle is a species. The first letter of haplotype name represent the marker: $\mathrm{i}$ for ITS2, $\mathrm{r}$ for $r b c \mathrm{~L}$ and $\mathrm{t}$ for $t r n \mathrm{~L}-\mathrm{F}$. Dots correspond with haplotype counts. 


\section{Morphometric analyses}

We gathered morphometric data from 436 herbarium samples and living plants of Aronia (including 187 samples of A. mitschurinii). We applied several methods of multivariate analyses using both 'classical' and geometric morphometrics approaches. As the dried herbarium samples might suffer from shrinking (Volkova et al. 2011), we compared the fresh and the dried herbarium samples of A. mitschurinii to calculate the shrinking coefficient. This coefficient was less than $4 \%$, therefore shrinking was not taken into account in our geometric morphometrics analyzes.

Several leaf-related morphological characters were measured, including 'classic' petiole and leaf length, leaf maximal width and position of maximal width. In geometric morphometrics analyses, we used the standard thin plate spline approach (Zelditch et al. 2012) with 10 landmarks located on the tip and base of the leaf plus on the points of maximal curvature on the leaf contour (see Fig. 7 for the examples). Coordinates of the landmarks were written to the data file with tpsDig (Rohlf 2010).

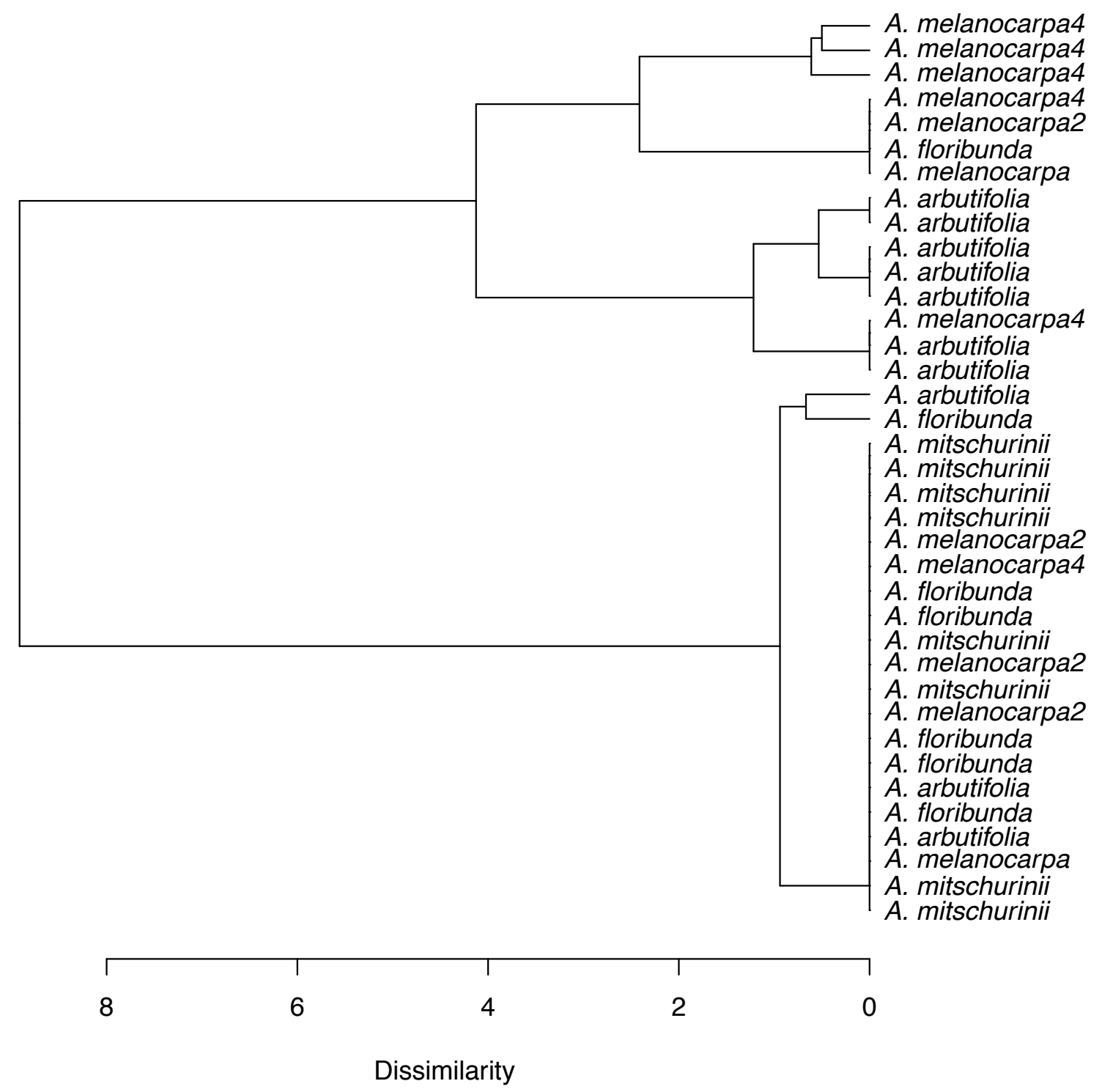

Fig. 3. Cluster analysis (binary distance, Ward clustering method) of the occurrence of eight basic $r b c \mathrm{~L}$ and ITS2 haplotypes. 
Consensus configuration, values of principal relative and partial warps (which characterize the degree of differences between the specimen and consensus configuration), were calculated with the $\mathrm{R}$ geomorph package (Adams \& Otarola-Castillo 2013) which implements TPS analysis in a way similar to principal component analysis (PCA). Principal component analysis of the relative warps matrix was employed for the classification of leaf shapes, similarly to the way described elsewhere (Shipunov \& Bateman 2005).

Many statistical methods have the ability to combine different types of data, and we used this to study the joint matrix that includes morphological characters and haplotype data of exactly the same samples (similar to Shipunov et al. 2004). We employed model-based clustering (Scrucca et al. 2016) and t-SNE algorithm (t-SNE is 't-Distributed Stochastic Neighbor Embedding') (van der Maaten \& Hinton 2008). The latter is a nonlinear dimensionality reduction algorithm, popular for representing high-dimensional data. More simple clustering of haplotype occurrence data used binary distances and Ward linkage. All statistical analyses were performed in R (R Core Team 2018).

\section{Results}

\section{DNA sequencing and molecular analysis}

Most of the analyses were based on ITS2 and $r b c$ L alignments. Analysis of the trnL-F and LEAFY data was more problematic, since they did not amplify well from many of the studied herbarium samples;

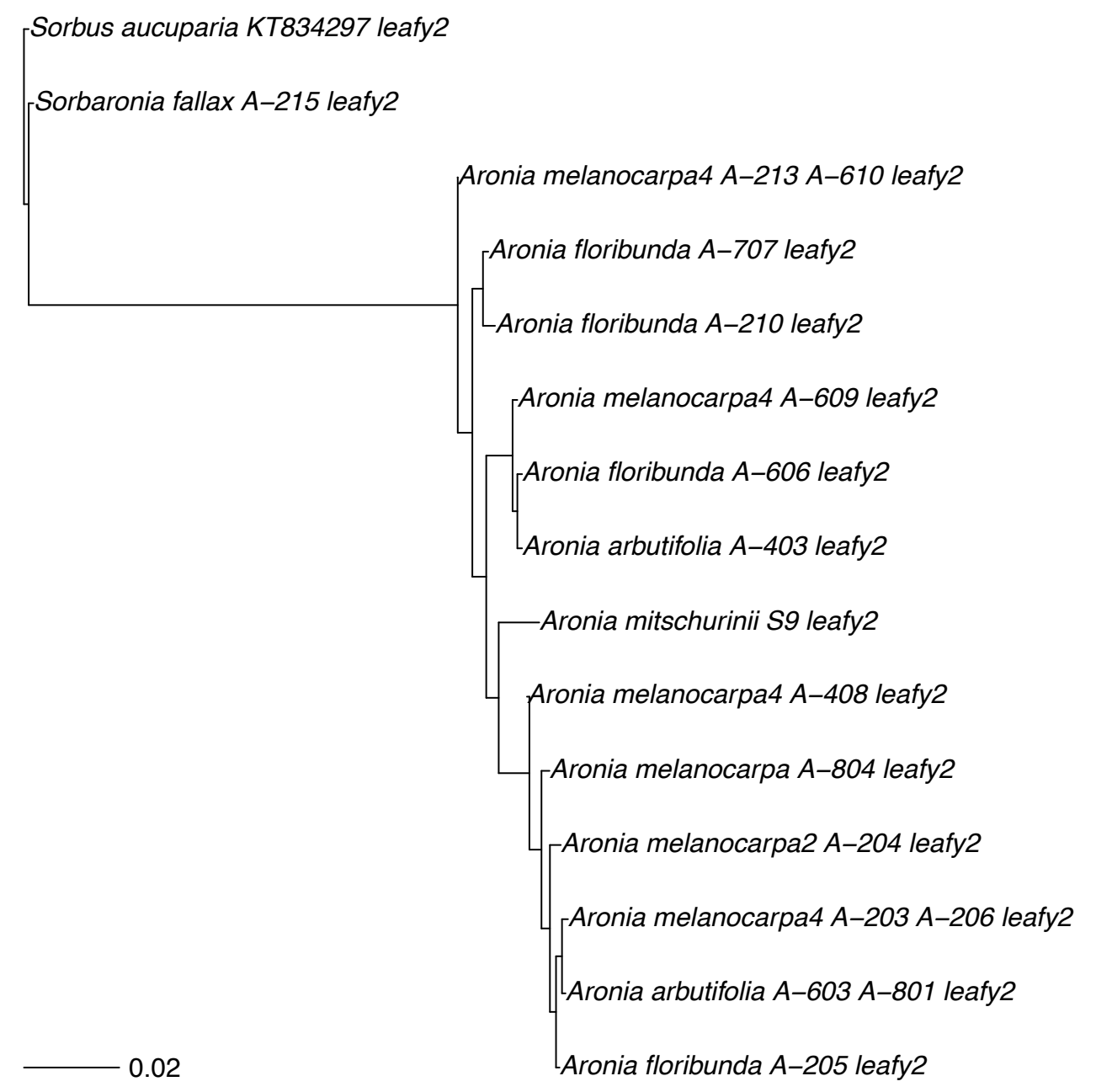

Fig. 4. ML tree from the LEAFY2 sequences. 
therefore, these markers were analyzed separately. The resulting trees always had extremely short branches (Fig. 1). Bootstrap was high enough (>97\%) to support only the whole Aronia group. Within Aronia, there were no clades receiving high statistical support.

Within each locus, most of our sequences differ only by several nucleotides. Across all sequences analyzed, we found ten haplotypes.

We counted only three haplotypes from $A$. mitschurinii samples whereas the more diverse sets were found in A. melanocarpa and A. arbutifolia (Fig. 2). As our phylogeny trees are not highly informative, we employed the cluster analysis of the haplotype occurrence (Fig. 3) which revealed the tendency of A. arbutifolia samples to group in one cluster ( 7 out of 10 are in one group). Aronia mitschurinii samples did not form a cluster, and grouped together with the bulk of other Aronia. Data from our trnL-F sequences were generally in agreement with $r b c \mathrm{~L}$ data. Only two haplotypes were found, one the most frequent and the other specific to A. arbutifolia.

We were able to sequence both variants (potential paralogs) of LEAFY second intron (Burgess et al. 2015), and therefore constructed two separate datasets, one for each variant. From $\times$ Sorbaronia fallax, we amplified the second copy, which was identical to the same copy of Sorbus aucuparia (GenBank ID KT834297). Each LEAFY dataset contained at least one A. mitschurinii sample. In the analyses of both

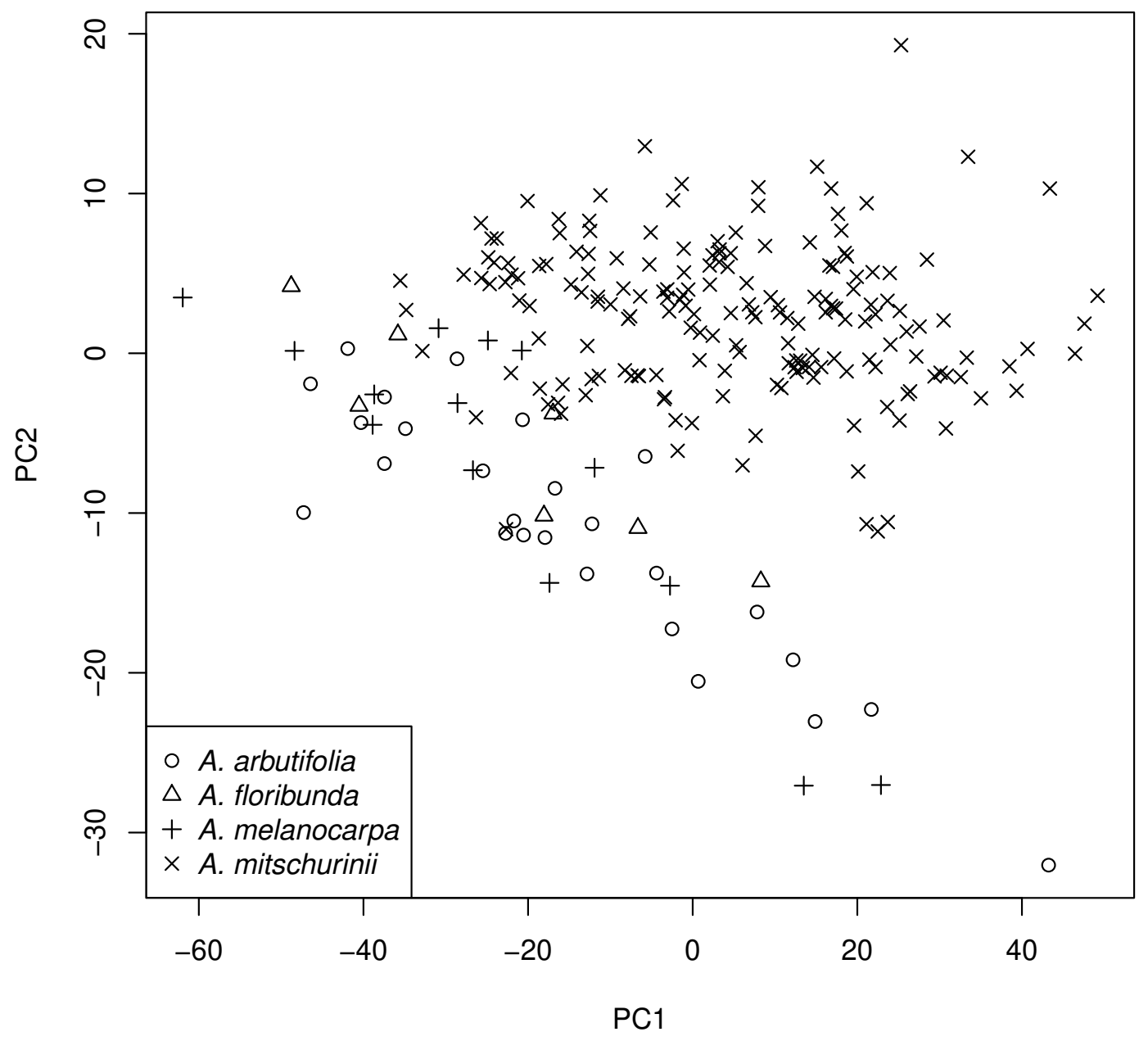

Fig. 5. Ordination on the plane of first two principal components from the analysis of morphological data. 
datasets, A. mitschurinii samples always robustly grouped with $A$. melanocarpa and did not group with Sorbus. This pattern is illustrated on Fig. 4 which reflects relationships within the second, most samplerich LEAFY dataset.

\section{Morphometric and combined analyses}

The principal component analysis (PCA) of morphometric data (Fig. 5) was able to clearly separate the A. mitschurinii samples. Samples of other species did not form clusters.

In the case of joint matrix (morphological characters + haplotype data), the scarcity of A. mitschurinii data did not allow for robust conclusions, but it is remarkable that these two A. mitschurinii samples occupy a separate branch on the minimum spanning tree and therefore distinctive (Fig. 6) from all other Aronia.

Geometric morphometrics was first used to produce the consensus shapes for each of our species. These shapes demonstrated the bending required to make imaginary thin plate splines to converge. Figure 7 shows that $A$. mitschurinii samples require the smaller deformations, whereas $A$. arbutifolia

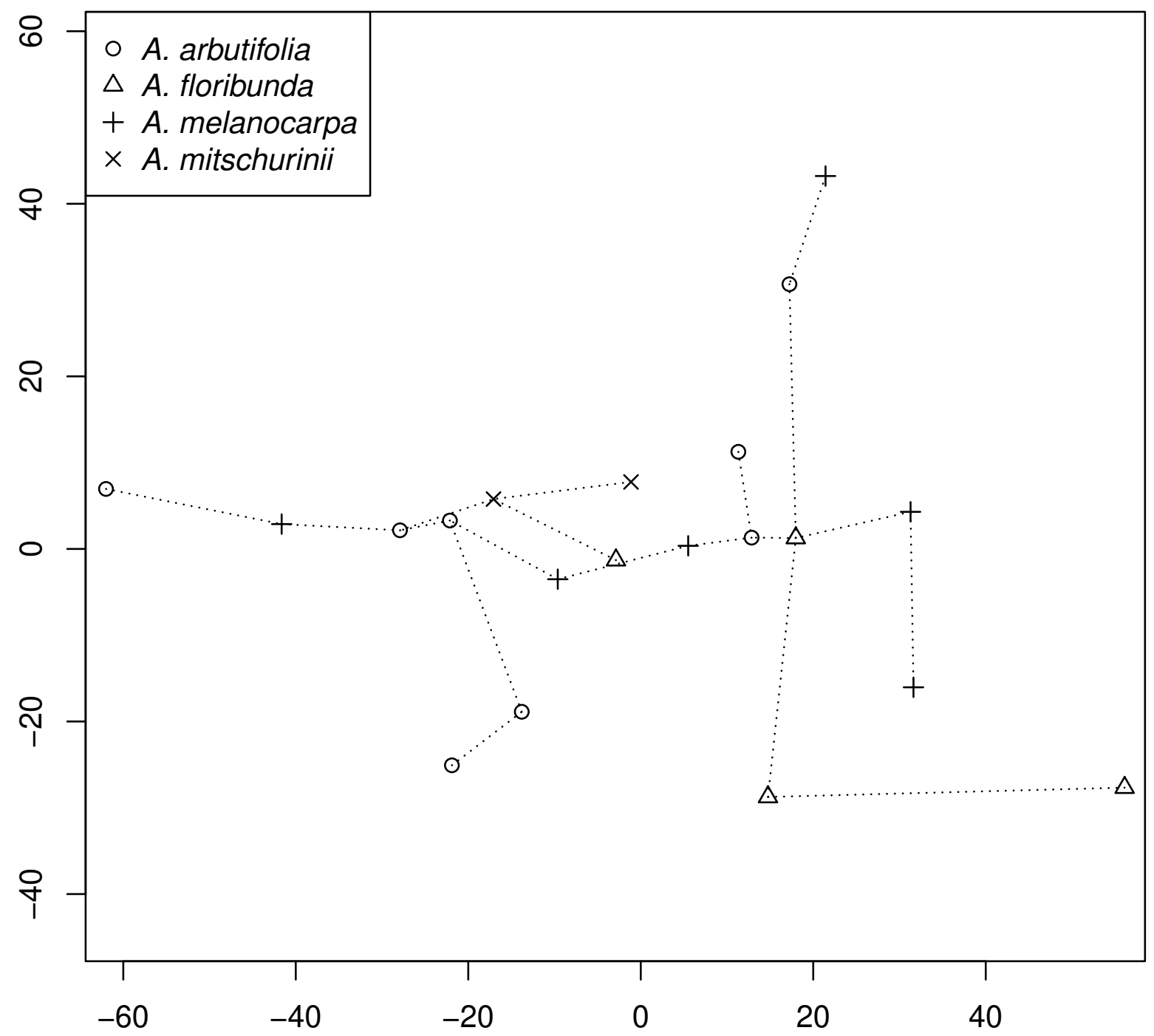

Fig. 6. Ordination from multidimensional scaling of the Gower distance matrix from the combined dataset of haplotypes occurrence and morphology. 
and A. floribunda require more modifications of their average shapes. PCA ordination of relative warps provided another view on the diversity of our samples. It was similar to the morphology ordination, and separated A. mitschurinii whereas other species were intermixed (Fig. 8).

We also tried two combined approaches which included geometric morphometrics data together with (1) morphology and (2) morphology + haplotype data from the same samples. PCA was not really helpful for the analysis of geometric morphometrics + morphology data, but t-SNE (van der Maaten \& Hinton 2008) allowed to arrange the samples in more understandable way (Fig. 9); here most of A. mitschurinii samples were clearly separated from the rest of Aronia. On the other hand, the model-based clustering (Scrucca et al. 2016) returned the clustering model with only one component (i.e., no internal clusters).

\section{Discussion}

Our phylogenetic analyses show that the overall genetic and morphological diversity within Aronia is low, and no clear species boundaries could be detected based on DNA data. In our morphological

A. arbutifolia

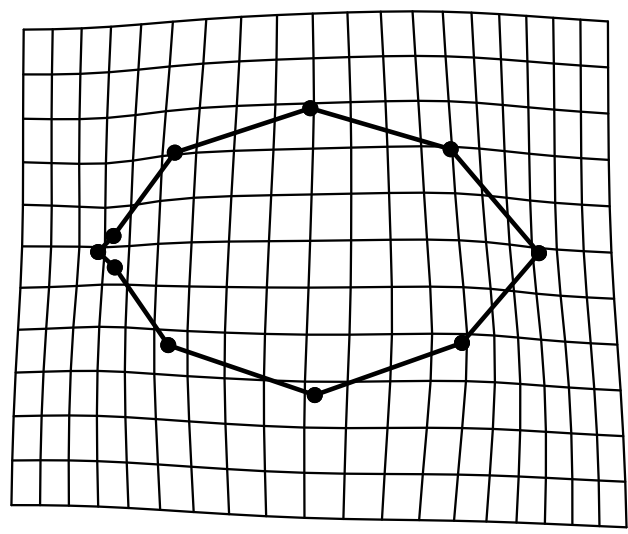

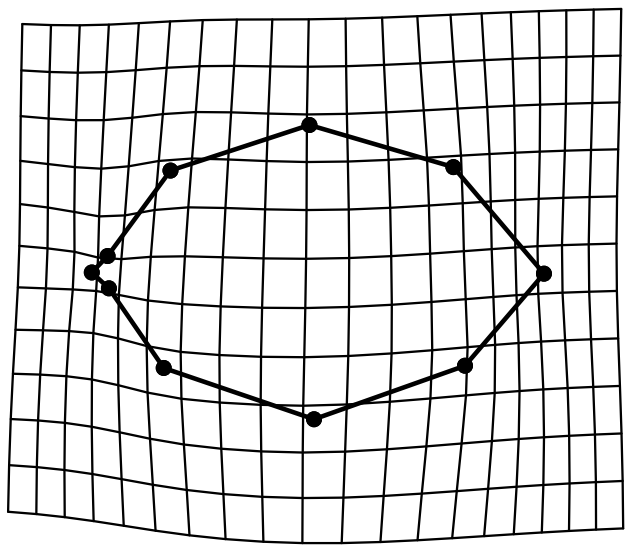

\section{A. floribunda}

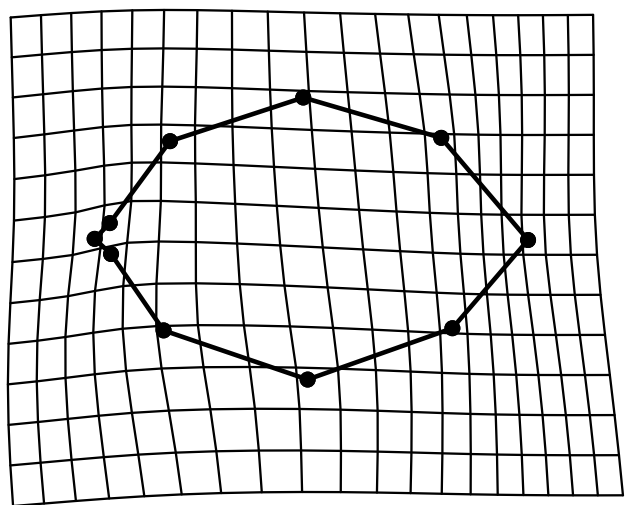

Fig. 7. Transformation grids of leaf shape from four Aronia species, with deformations required to reach the overall average shape. 
and combined analyses, only A. mitschurinii could be separated with confidence. These results are in contrast to Connolly (2014) where four Aronia species could be morphologically distinguished.

This discrepancy between morphological and molecular data could mean that Aronia still awaits more advanced methods of data collection and data analysis. However, considering the diversity of our approaches which include phylogeny trees, haplotype analysis, classic and geometric morphometry and combined integrative analysis, the possible conclusion is that at least some borders within Aronia are not expressed as it is typical in neighboring genera (Guo et al. 2012, Li et al. 2012) but may delimit only subspecific or lower level variation; it is even possible that most of the Aronia samples represent one polymorphic species.

No clear similarities, morphological or genetic, between any Aronia and Sorbus aucuparia were discovered. The $\times$ Sorbaronia fallax sample has the ITS2 and second copy of LEAFY second intron identical to Sorbus aucuparia, indicative of a hybridization event between Sorbus aucuparia and Aronia melanocarpa. However, this pattern was not present in our samples of A. mitschurinii. Therefore, we believe that the hybrid origin of $A$. mitschurinii is under question. This hypothesis has an independent support from observations on $A$. mitschurinii propagation which almost always results in the uniform progeny (Vinogradova \& Kuklina 2014). In addition, whereas morphology alone allows for the separation

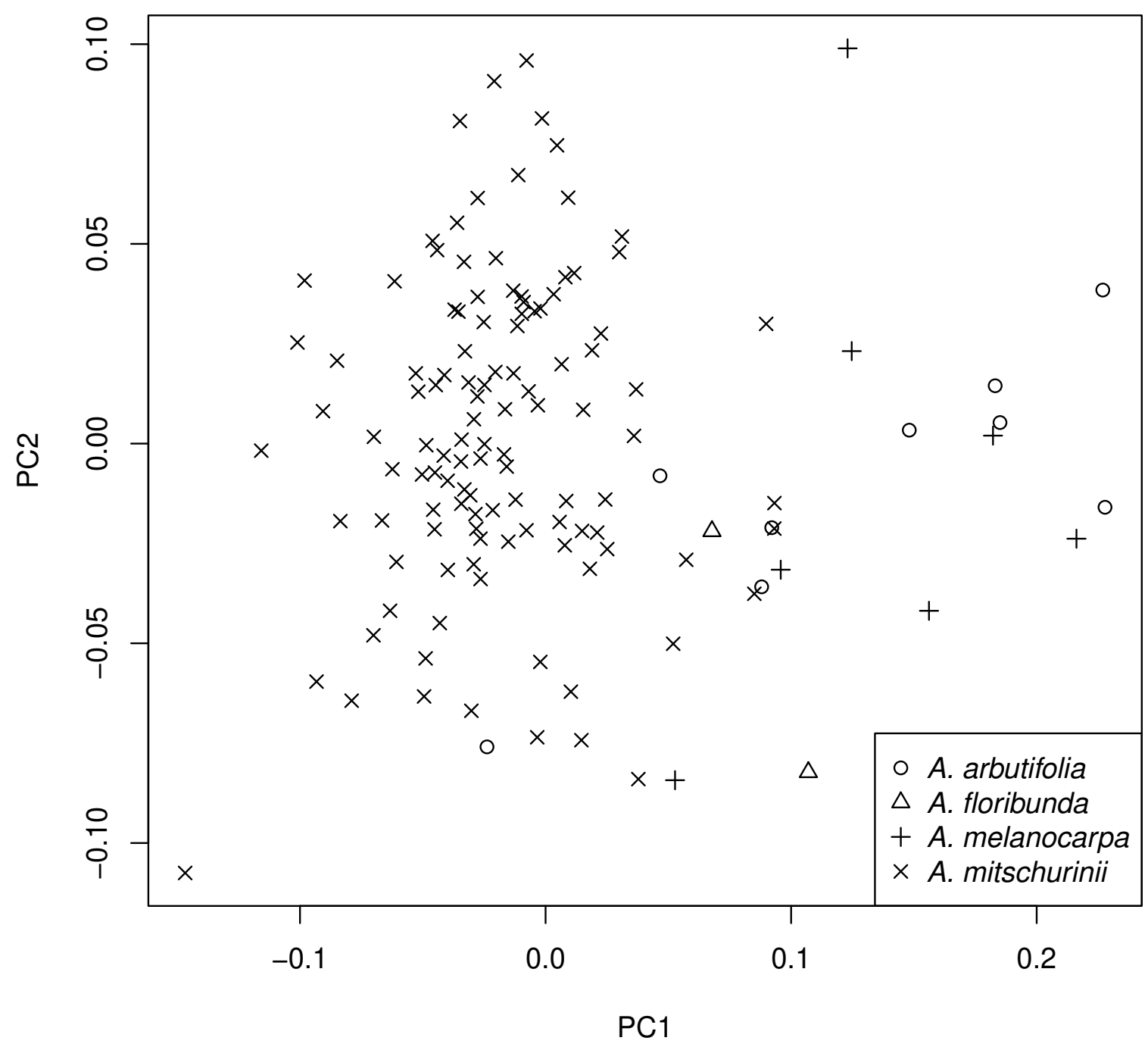

Fig. 8. Ordination from the principal component analysis of relative warps. 
of $A$. mitschurinii from other species, there is no support of such dissimilarity from molecular data. DNA-wise, A. mitschurinii does not differ from the other Aronia species.

The apparent conflict between AFLP (Leonard et al. 2013) and our sequencing analysis could be due to over-sensitivity of the first analysis (Pelser et al. 2003). It is important to note that Leonard et al. (2013) did not show the affinity of A. mitschurinii to the Sorbus, they have had only found similarities with $\times$ Sorbaronia hybrids.

It is also possible that A. mitschurinii is a backcross to Aronia, and the Sorbus genome is largely lost. Therefore, we cannot fully exclude the possibility that thorough cloning of multiple markers or highthroughput sequencing, might reveal the traces of others genomes in A. mitschurinii. Thus, the next step in Aronia phylogeny research should involve more high resolution molecular markers, especially multilocus nuclear data.

\section{Acknowledgements}

We are grateful to the curators of CAS, HUH, JEPS, MHA, MO, NY, UC and US herbaria for the kind permission to analyze herbarium samples. Aronia samples from Russia were collected mostly around

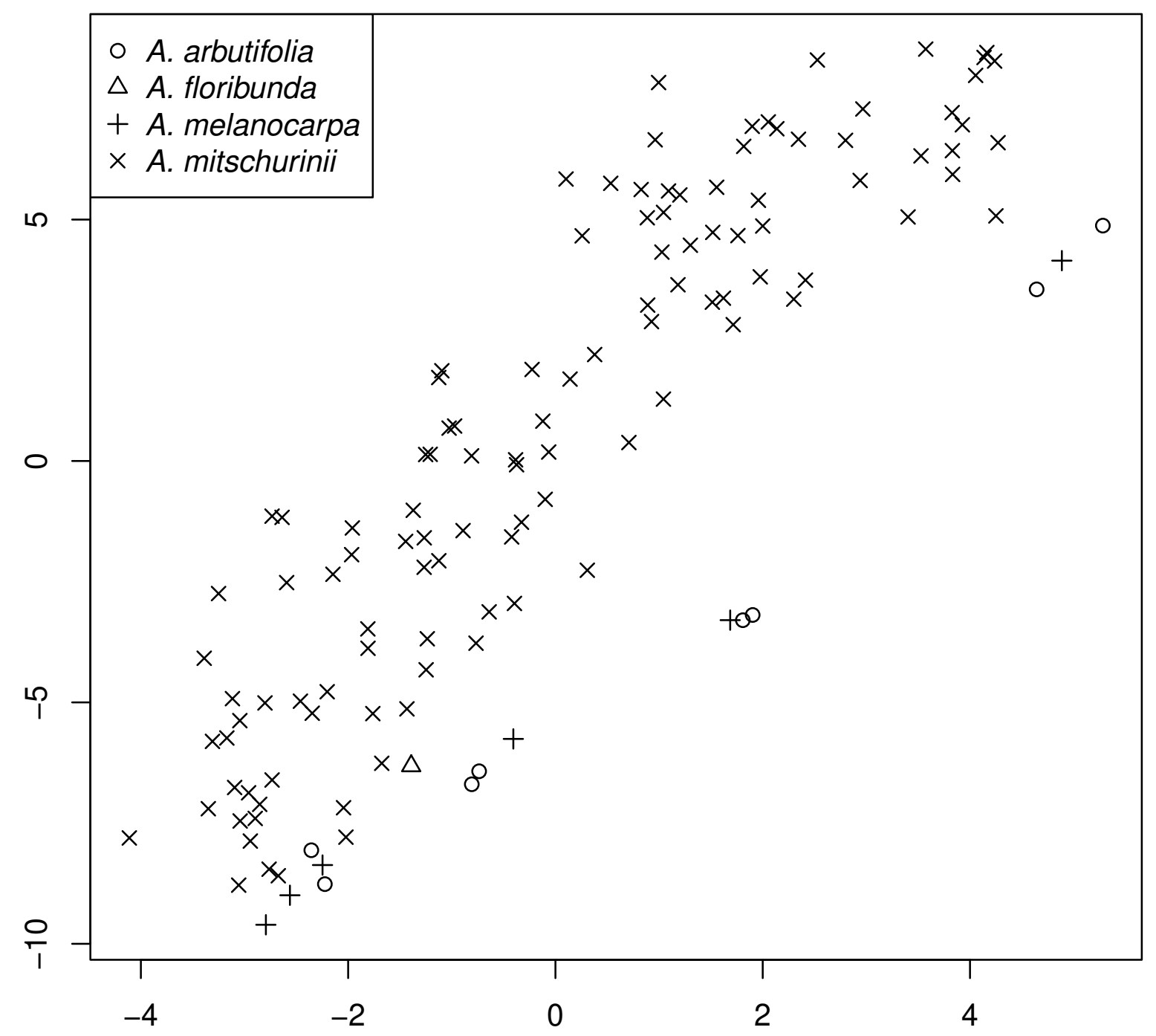

Fig. 9. t-SNE ordination of the combined morphology + geometric morphometrics data. 
'Lake Moldino' biological station, and we thank the station staff for the assistance. We thank the College of Art and Sciences and the Department of Biology of Minot State University for the financial support. From May 2014, this research is supported by North Dakota INBRE.

\section{References}

Adams D.C. \& Otarola-Castillo E. 2013. geomorph: an R package for the collection and analysis of geometric morphometric shape data. Methods in Ecology and Evolution 4: 393-399.

https://doi.org/10.1111/2041-210X.12035

Brand M.H. 2010. Aronia: native shrubs with untapped potential. Arnoldia 67: 14-25.

Burgess M.B., Cushman K.R., Doucette E.T., Frye C.T. \& Campbell C.S. 2015. Understanding diploid diversity: A first step in unraveling polyploid, apomictic complexity in Amelanchier. American Journal of Botany 102: 2041-2057. https://doi.org/10.3732/ajb.1500330

Campbell C.S., Evans R.C., Morgan D.R., Dickinson T.A. \& Arsenault M.P. 2007. Phylogeny of subtribe Pyrinae (formerly the Maloideae, Rosaceae): limited resolution of a complex evolutionary history. Plant Systematics and Evolution 266: 119-145. https://doi.org/10.1007/s00606-007-0545-y

Connolly B.A. 2009. $\times$ Sorbaronia fallax (Rosaceae): A new record of an intergeneric hybrid in Connecticut. Rhodora 111: 123-125. https://doi.org/10.3119/08-23.1

Connolly B.A. 2014. Collection, description, taxonomic relationships, fruit biochemistry, and utilization of Aronia melanocarpa, A. arbutifolia, A. prunifolia, and A. mitschurinii. PhD thesis. Paper 342. University of Connecticut.

Dluzewska J., Slesak I. \& Kruk J. 2013. Molecular analysis of Sorbus sp. from the Pieniny Mts. and its relation to other Sorbus species. Acta Biologica Cracoviensia, Series Botanica 55: 86-92.

https://doi.org/10.2478/abcsb-2013-0009

Guo W., Yu Y., Shen R.J., Liao W.B., Chin S.W. \& Potter D. 2011. A phylogeny of Photinia sensu lato (Rosaceae) and related genera based on nrITS and cpDNA analysis. Plant Systematics and Evolution 291: 91-102. https://doi.org/10.1007/s00606-010-0368-0

Hardin J.W. 1973. The enigmatic chokeberries. Bulletin of the Torrey Botanical Club 100: 178-184. https://doi.org/10.2307/2484630

Kask K. 1987. Large-fruited black chokeberry (Aronia melanocarpa). Fruit Varieties Journal 41: 47.

Kuzmina M. \& Ivanova N. 2011. Primer sets for plants and fungi. Available from:

http://ccdb.ca/site/wp-content/uploads/2016/09/CCDB_PrimerSets-Plants.pdf [accessed 19 Jun. 2019].

Larsson A. 2014. AliView: a fast and lightweight alignment viewer and editor for large data sets. Bioinformatics 30: 3276-3278. https://doi.org/10.1093/bioinformatics/btu531

Leonard P.J. 2011. Aronia mitschurinii: solving a horticultural enigma. MA thesis. Paper 183. University of Connecticut.

Leonard P.J., Brand M.H., Connolly B.A. \& Obae S.G. 2013. Investigation of the origin of Aronia mitschurinii using amplified fragment length polymorphism analysis. HortScience 48: 520-524.

https://doi.org/10.21273/HORTSCI.48.5.520

Li Q.Y., Guo W., Liao W.B., Macklin J.A. \& Li J.H. 2012. Generic limits of Pyrinae: insights from nuclear ribosomal DNA sequences. Botanical Studies 53: 151-164.

Li M., Ohi-Toma T., Gao Y.D., Xu B., Zhu Z.M., Ju W.B. \& Gao X.F. 2017. Molecular phylogenetics and historical biogeography of Sorbus sensu stricto (Rosaceae). Molecular Phylogenetics and Evolution 111: 76-86. https://doi.org/10.1016/j.ympev.2017.03.018 
Linnaeus C. 1753. Species Plantarum 1: 477. Holmiae.

Lo E.Y. \& Donoghue M.J. 2012. Expanded phylogenetic and dating analyses of the apples and their relatives (Pyreae, Rosaceae). Molecular Phylogenetics and Evolution 63: 230-243.

https://doi.org/10.1016/j.ympev.2011.10.005

Lo E.Y., Stefanović S. \& Dickinson T.A. 2007. Molecular reappraisal of relationships between Crataegus and Mespilus (Rosaceae, Pyreae) - two genera or one? Systematic Botany 32: 596-616.

https://doi.org/10.1600/036364407782250562

Maaten L. van der \& Hinton G. 2008. Visualizing data using t-SNE. Journal of Machine Learning Research 9: 2579-2605.

Medikus F.K. 1789. Philosophische Botanik mit kritischen Bemerkungen: 140. Mannheim.

Oh S.H. \& Potter D. 2003. Phylogenetic utility of the second intron of LEAFY in Neillia and Stephanandra (Rosaceae) and implications for the origin of Stephanandra. Molecular Phylogenetics and Evolution 29: 203-215. https://doi.org/10.1016/S1055-7903(03)00093-9

Paradis E., Claude J. \& Strimmer K. 2004. APE: analyses of phylogenetics and evolution in R language. Bioinformatics 20: 289-290. https://doi.org/10.1093/bioinformatics/btg412

Pelser P.B., Gravendeel B. \& Meijden R van der. 2003. Phylogeny reconstruction in the gap between too little and too much divergence: the closest relatives of Senecio jacobaea (Asteraceae) according to DNA sequences and AFLPs. Molecular Phylogenetics and Evolution 29: 613-628.

https://doi.org/10.1016/S1055-7903(03)00139-8

Persson Hovmalm H.A., Jeppsson N., Bartish I.V. \& Nybom H. 2004. RAPD analysis of diploid and tetraploid populations of Aronia points to different reproductive strategies within the genus. Hereditas 141: 301-312. https://doi.org/10.1111/j.1601-5223.2004.01772.x

R Core Team. 2018. R: A language and environment for statistical computing. R Foundation for Statistical Computing, Vienna, Austria. Available from http://www.R-project.org/ [accessed 1 Jan. 2018].

Robertson K.R., Phipps J.B., Rohrer J.R. \& Smith P.G. 1991. A synopsis of genera in Maloideae (Rosaceae). Systematic Botany 16: 376-394. https://doi.org/10.2307/2419287

Rohlf F.J. 2010. tpsDig. Version 2.16. State University at Stony Brook, N.Y. Available from http://life.bio.sunysb.edu/morph [accessed 20 Feb. 2014].

Schliep K.P. 2011. phangorn: phylogenetic analysis in R. Bioinformatics 27: 592-593. https://doi.org/10.1093/bioinformatics/btq706

Schneider C.K. 1906. Species varietatesque Pomacearum novae. Repertorium Specierum Novarum Regni Vegetabilis 3: 134.

Scrucca L., Fop M., Murphy T.B. \& Raftery A.E. 2016. mclust 5: clustering, classification and density estimation using Gaussian finite mixture models. The R Journal 8: 205-233.

Sennikov A.N. \& Phipps J.B. 2013. Atlas Florae Europaeae notes, 19-22. Nomenclatural changes and taxonomic adjustments in some native and introduced species of Malinae (Rosaceae) in Europe. Willdenowia 43: 33-44. https://doi.org/10.3372/wi.43.43104

Shipunov A. \& Bateman R. 2005. Geometric morphometrics as a tool for understanding Dactylorhiza (Orchidaceae) diversity in European Russia. Biological Journal of the Linnean Society 85: 1-12. https://doi.org/10.1111/j.1095-8312.2005.00468.x

Shipunov A., Fay M.F., Pillon Y., Bateman R.M. \& Chase M.W. 2004. Dactylorhiza (Orchidaceae) in European Russia: combined molecular and morphological analysis. American Journal of Botany 91: 1419-1427. https://doi.org/10.3732/ajb.91.9.1419 
Skvortsov A.K. \& Majtulina J.K. 1982. On the diferences of cultivated black-fruited Aronia from its wild ancestors. Bulletin of Main Botanical Garden 126: 35-40. [In Russian.]

Skvortsov A.K., Majtulina J.K. \& Gorbunov J.N. 1983. On the place, time and putative way of the cultivated black-fruited Aronia origin. Bulletin of the Moscow Society of Naturalists. Biological Series 88: 88-96. [In Russian.]

Smolik M., Ochmian I. \& Smolik B. 2011. RAPD and ISSR methods used for fingerprinting selected, closely related cultivars of Aronia melanocarpa. Notulae Botanicae Horti Agrobotanici Cluj-Napoca 39: 276-284. https://doi.org/10.15835/nbha3926268

Sun J., Shi S., Li J., Yu J., Wang L., Yang X., Guo L. \& Zhou S. 2018. Phylogeny of Maleae (Rosaceae) based on multiple chloroplast regions: implications to genera circumscription. BioMed Research International 2018: 7627191. https://doi.org/10.1155/2018/7627191

Taheri R., Connolly B.A., Brand M.H. \& Bolling B.W. 2013. Underutilized chokeberry (Aronia melanocarpa, Aronia arbutifolia, Aronia prunifolia) accessions are rich sources of anthocyanins, flavonoids, hydroxycinnamic acids, and proanthocyanidins. Journal of Agricultural and Food Chemistry 61: 8581-8588. https://doi.org/10.1021/jf402449q

Thompson J.D., Gibson T.J., Plewniak F., Jeanmougin F. \& Higgins D.G. 1997. The CLUSTAL_X windows interface: flexible strategies for multiple sequence alignment aided by quality analysis tools. Nucleic Acids Research 25 (24): 4876-4882. https://doi.org/10.1093/nar/25.24.4876

Vinogradova Y.K. \& Kuklina A.G. 2014. Aronia mitschurinii: from Origination to Naturalization. GEOS, Moscow. [In Russian.]

Volkova P., Kasatskaya S., Boiko A. \& Shipunov A. 2011. Stability of leaf form and size during specimen preparation of herbarium specimens. Feddes Repertorium 121: 219-225.

https://doi.org/10.1002/fedr.201000021

Zarrei M., Stefanovic S. \& Dickinson T.A. 2014. Reticulate evolution in North American black-fruited hawthorns (Crataegus section Douglasia; Rosaceae): evidence from nuclear ITS2 and plastid sequences. Annals of Botany 114: 253-269. https://doi.org/10.1093/aob/mcu116

Zelditch M.L., Swiderski D.L. \& Sheets H.D. 2012. Geometric morphometrics for biologists: a primer. Academic Press.

Manuscript received: 26 August 2018

Manuscript accepted: 22 August 2019

Published on: 23 October 2019

Topic editor: Frederik Leliaert

Desk editor: Connie Baak

Printed versions of all papers are also deposited in the libraries of the institutes that are members of the EJT consortium: Muséum national d'Histoire naturelle, Paris, France; Meise Botanic Garden, Belgium; Royal Museum for Central Africa, Tervuren, Belgium; Royal Belgian Institute of Natural Sciences, Brussels, Belgium; Natural History Museum of Denmark, Copenhagen, Denmark; Naturalis Biodiversity Center, Leiden, the Netherlands; Museo Nacional de Ciencias Naturales-CSIC, Madrid, Spain; Real Jardín Botánico de Madrid CSIC, Spain; Zoological Research Museum Alexander Koenig, Bonn, Germany; National Museum, Prague, Czech Republic. 